\title{
MODIFICATION OF DOMINANCE BY SELECTION IN THE HOMOZYGOTE
}

\author{
JAMES N. THOMPSON, Jr., and J. M. THODAY \\ Department of Genetics, University of Cambridge, England
}

Received 7.i.72

\section{INTRODUCTION}

THE evolution of dominance has long been a controversial subject. Major theories (see O'Donald, 1968) involve either the wild type evolving dominance in response to the repeated appearance of deleterious mutations (Fisher, 1928, 1931), the heterozygote increasing in fitness and dominance evolving as an advantageous allele spreads through a population (Parsons and Bodmer, 1961), or dominance evolving in a polymorphism maintained by frequency dependent or disruptive selection (Sheppard, 1959, 1961). A fourth situation, that the degree of dominance could be increased through selection in the originally recessive homozygote, is seldom considered, though Sheppard (1962; Clarke and Sheppard, 1962) discussed this possibility. Modification of dominance, by moving the expression of the homozygote toward that of the heterozygote, has recently been investigated experimentally in the semi-dominant "Hairy wing" mutant of Drosophila melanogaster (Ohh and Sheldon, 1970) and may have occurred naturally in, for example, the crest form of domestic poultry (Fisher, 1935; Ohh and Sheldon, 1970). Other examples, such as selection for increased penetrance and expressivity affecting the level of dominance of the polydactyly mutant in mice (Bodmer, 1960), indicate that some degree of dominance modification might occasionally arise as an associated response during selection for expression modifiers. Selected lines developed originally in the course of studies of wing venation mutant modifiers in Drosophila melanogaster have allowed us to do confirmatory experiments by asking the question: What is the relationship between modifiers of dominance and modifiers of phenotypic expression?

\section{Methods}

The following mutants, arranged by chromosome map position, were used in this study: $s h v$ (short vein), 2-3.8, veins L2 and L4 do not reach the wing margin, while in later generations of selection L3 also became shortened slightly; ve (veinlet), 3-0.2, veins L2, L3, L4 and L5 shortened; $r i$ (radius incompletus), 3-47.0, vein L2 shortened; and $c i$ (cubitus interruptus), 4-0, vein L4 usually possesses one or more gaps. The Oregon stock was used as a standard wild-type stock, and in one phase of the study, shv lines were crossed to the second chromosome mutant $p x$ (plexus), which possesses a network of extra veins, particularly along the margins and tips of the long veins. For detailed descriptions see Lindsley and Grell (1967). The necessary wing nomenclature is shown in plate II, $a$.

Each of the four wing mutants was outcrossed to a newly caught wildtype strain to increase the genetic variability in the background after resegregation of the homozygous mutants. Then four replicate lines of each mutant 


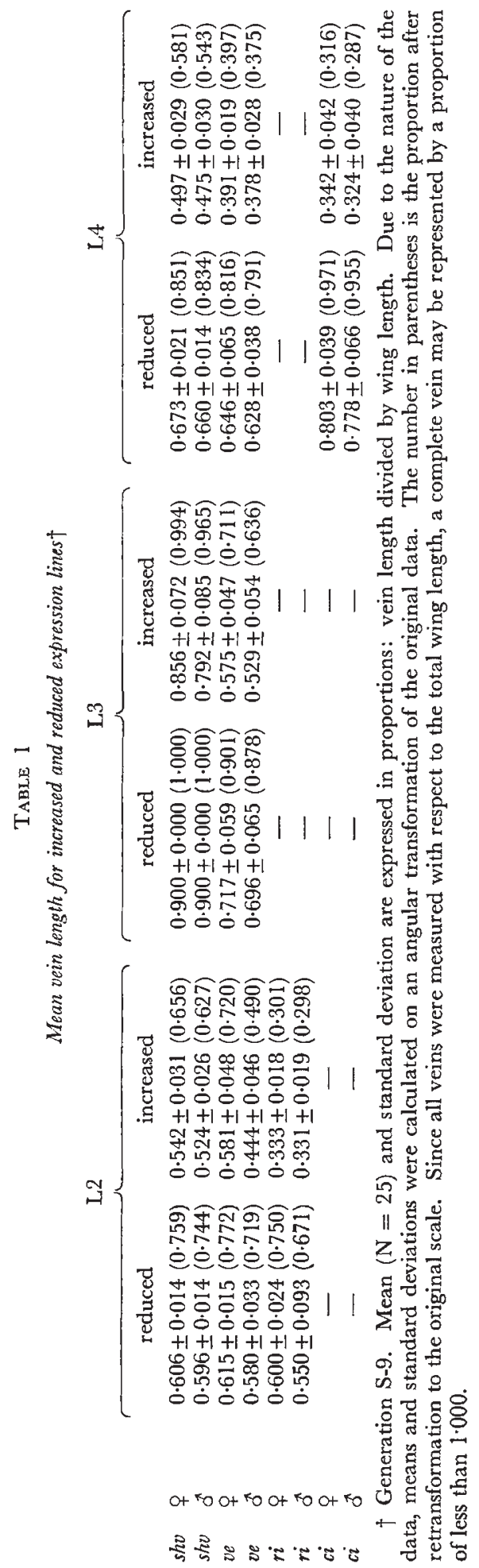


were selected at $25^{\circ} \mathrm{C}$. for increased, and four for reduced, expression of the shortened vein phenotypes (plate I). Selection was only exercised on veins L2, L3 and L4, and though the three long veins responded at different rates, by the ninth generation significant responses had been obtained for at least one of the veins affected in each mutant (table 1). Five replicate single-pair crosses were then made to produce double heterozygotes, both among the increased- and among the reduced-expression lines. Crosses were also made with a standard Oregon wild-type stock to produce single-mutant heterozygotes. Similar double and single heterozygote crosses from unselected mutant lines served as controls. Reciprocal crosses were made in each case. All offspring eclosing from each cross within 6 days were scored for the presence or absence of thin or shortened veins.

The sho line selected for reduced expression, i.e. selected for long veins, had a high frequency of flies with extra vein material in the marginal, submarginal and discal cells (plate 1,a). For this reason, shv extra-vein lines, $s h v$ short (increased expression) lines, $p x$, and Oregon wild type were crossed in all combinations to observe whether the same relationships found for vein gaps also held for extra vein material. Offspring eclosing from each of five replicates of each cross were scored for the presence or absence of extra material or of gaps in the vein. Gaps, however, were never observed in this series of crosses.

\section{Results}

\section{(i) Vein gaps in heterozygotes}

Dominance is a term used to describe the relation between a pair of alleles and their phenotypic resultant. In the discussion that follows, since all mutants used are complete recessives, dominance will be said to have been modified if the heterozygote fails to resemble the originally dominant homozygote to any degree in a significant number of individuals examined. Sixty-eight crosses among selected, original stock, Oregon wild type, and plexus lines were made, from each of which about $400 \mathrm{~F}_{1}$ offspring were scored. As the principal effect of each of the four selected mutants is to shorten one or more longitudinal veins, throughout this discussion the terms "long" and "short" will be used to designate reduced expression and increased expression lines, respectively.

Of the six double heterozygotes eclosing from crosses of original stock lines, only two combinations produced $F_{1}$ vein abnormalities. In one of these control cross combinations, shv/ + ; $v /+$, one female from 820 offspring possessed a shortened L5 vein (plate II, $f$ ). This is a common abnormality occuring at a low frequency in many crosses involving the ve mutant. Eight of 892 sho/ + ; ril + offspring had a small terminal gap in the L2 vein (plate II, $e$ ), though the difference between this and the comparable double heterozygote offspring from the cross involving "long" lines, in which none of 621 flies possessed vein abnormalities, is not statistically significant. In the same cross using $s h v$ and $r i$ " short " lines, however, 151 of a total of 738 offspring had terminal gaps in the L2 veins, highly significantly different from the "long" and stock control crosses (tables 2 and 3).

Similar highly significant differences were obtained in every other comparison of "short"-line-derived versus "long"-line-derived double 
TABLE 2

Comparison of vein gap appearance in short $\times$ short and in long $\times$ long crosses. Chi-squares are for one degree of freedom

\begin{tabular}{|c|c|c|c|c|c|c|}
\hline \multirow[b]{2}{*}{ Cross } & \multicolumn{2}{|c|}{ Short $\times$ Short } & \multicolumn{2}{|c|}{ Long $\times$ Long } & \multirow[b]{2}{*}{$\mathrm{N}$} & \multirow[b]{2}{*}{$\chi^{2}$} \\
\hline & + & gaps & + & gaps & & \\
\hline$s h v \times v e$ & 281 & 109 & 371 & 0 & 761 & $121 \cdot 02$ \\
\hline$v e \times s h v$ & 366 & 73 & 260 & 1 & 700 & $45 \cdot 69$ \\
\hline$s h v \times r i$ & 279 & 85 & 318 & 0 & 682 & $84 \cdot 83$ \\
\hline$r i \times s h v$ & 308 & 66 & 303 & 0 & 677 & $59 \cdot 25$ \\
\hline$s h v \times c i$ & 397 & 27 & 430 & 0 & 854 & $28 \cdot 28$ \\
\hline$c i \times s h v$ & 515 & 13 & 492 & 0 & 1020 & $12 \cdot 27$ \\
\hline$v e \times r i$ & 181 & 138 & 365 & 1 & 685 & 194.73 \\
\hline$r i \times v e$ & 258 & 145 & 293 & 0 & 696 & $133 \cdot 16$ \\
\hline$v e \times c i$ & 308 & 188 & 382 & 39 & 917 & $100 \cdot 27$ \\
\hline$c i \times v e$ & 323 & 97 & 317 & 8 & 745 & $64 \cdot 43$ \\
\hline$r i \times c i$ & 440 & 68 & 391 & 0 & 899 & $56 \cdot 62$ \\
\hline$c i \times r i$ & 356 & 3 & 237 & 1 & 597 & 一* \\
\hline
\end{tabular}

heterozygotes, with the exception of the $c i$ i $\times r i$ cross. The reciprocal cross $\left(r i q \times c i \delta^{\top}\right)$ results were significantly different (table 2$)$, possibly indicating the sex-linkage of one set of factors.

It should be noted that the phenotypes of the double heterozygotes are in no way similar to those of double homozygotes. The heterozygotes possess rather small gaps or thin areas, most often in veins affected by both of the mutants involved (plate II, $g, h$ ). Double homozygotes, on the other hand, are generally additive (plate II, $b-d)$.

Since the criterion for modification of dominance is that the phenotype of the $\mathrm{F}_{1}$ must be significantly different from the originally dominant homozygote in some degree of phenotypic expression, these results show that selection in the homozygote for enhancing the phenotype has an effect on dominance relationships in the $F_{1}$. Since in each case described thus far two major genes that affect venation have been present in each cross, a series of crosses were made to determine whether similar results would be found in the presence of a single mutant. One set of crosses between "short" lines and Oregon wild type resulted in vein interruptions in the $F_{1}$. Of $700 F_{1}$ offspring from the cross of ve " short" to Oregon, 97 possessed shortened L5 veins (plate II, $f$ ). This is significantly different from the similar crosses involving ve "long" lines (table 3) and indicates that selection in the

TABLE 3

Comparison of gap appearance in crosses of selected lines ( $L$, long; $S$, short) to mutant stock $(\$)$ and Oregon wild type

\begin{tabular}{|c|c|c|c|c|c|c|c|c|c|}
\hline \multicolumn{2}{|c|}{ Cross A } & \multicolumn{2}{|c|}{ Cross B } & \multicolumn{2}{|c|}{ Cross A } & \multicolumn{2}{|c|}{ Cross B } & \multirow[b]{2}{*}{$\mathbf{N}$} & \multirow[b]{2}{*}{$\chi^{2}$} \\
\hline 우 & $\sigma$ & 우 & o & + & gaps & + & gaps & & \\
\hline$s h v \$$ & $r i \$$ & $s h v \mathrm{~L}$ & $r i \mathrm{~L}$ & 536 & 3 & 318 & 0 & 857 & _* \\
\hline$r i \$$ & $s h v \$$ & $r i \mathrm{~L}$ & $s h v \mathbf{L}$ & 348 & 5 & 303 & 0 & 656 & -* \\
\hline$s h v \$$ & $r i \$$ & $s h v \mathrm{~S}$ & $r i \mathrm{~S}$ & 536 & 3 & 279 & 85 & 903 & $128 \cdot 36$ \\
\hline$r i \$$ & $s h v \$$ & $r i \mathrm{~S}$ & $s h v \mathrm{~S}$ & 348 & 5 & 308 & 66 & 727 & $54 \cdot 29$ \\
\hline ve $\mathrm{S}$ & Oregon & ve $\mathrm{L}$ & Oregon & 247 & 54 & 340 & 2 & 643 & $60 \cdot 65$ \\
\hline Oregon & ve $\mathrm{S}$ & Oregon & ve $\mathrm{L}$ & 356 & 43 & 479 & 0 & 878 & $54 \cdot 28$ \\
\hline
\end{tabular}

* Not statistically significant. 
homozygote has increased the degree of dominance of veinlet to an extent that makes it semi-dominant for the expression of L5 gaps.

\section{(ii) Extra vein material in heterozygotes}

All processes of vein formation, including plexation and gap production, appear to be closely related in development. Selection for reduced expression of the shv phenotype resulted in a large number of individuals carrying extra vein material, particularly in the marginal, submarginal and discal cells (plate I, a). The wild-type vein is dominant to both vein gap and extra vein phenotypes. Thus experiments comparing the appearance of extra vein material in crosses with the shv "long" lines (increased expression with respect to extra veins) versus those involving shv "short" lines (decreased expression in this context) are formally parallel to those described above for vein gaps, with only the terminology reversed. Though crosses of $p x$ stock or shv "long " lines to wild type produced only an occasional fly with extra vein material (table 4 ), the double heterozygote $s h v+1+p x \mathrm{~F}_{1}$ was made

TABLE 4

Comparisons of extra vein appearance in crosses involving short and long shv, plexus and Oregon wild-type lines

\begin{tabular}{|c|c|c|c|c|c|c|c|c|c|}
\hline \multicolumn{2}{|c|}{ Cross A } & \multicolumn{2}{|c|}{ Cross B } & \multicolumn{2}{|c|}{ Cross A } & \multicolumn{2}{|c|}{ Cross B } & \multirow[b]{2}{*}{$\mathrm{N}$} & \multirow[b]{2}{*}{$\chi^{2}$} \\
\hline 우 & व & 우 & क & + & $\begin{array}{l}\text { extra } \\
\text { veins }\end{array}$ & + & $\begin{array}{c}\text { extra } \\
\text { veins }\end{array}$ & & \\
\hline$p x$ & $\operatorname{shv} \mathbf{L}$ & $p x$ & $s h v \mathrm{~S}$ & 69 & 255 & 318 & 8 & 650 & $392 \cdot 18$ \\
\hline $\operatorname{sh} v \mathbf{L}$ & $p x$ & $s h v \mathrm{~S}$ & $p x$ & 134 & 249 & 427 & 0 & 810 & $400 \cdot 82$ \\
\hline$p x$ & $\operatorname{shv} \mathbf{L}$ & Oregon & $\operatorname{shv} \mathrm{L}$ & 69 & 255 & 417 & 0 & 741 & $500 \cdot 40$ \\
\hline shv $\mathrm{L}$ & $p x$ & $\operatorname{shv} \mathbf{L}$ & Oregon & 134 & 249 & 386 & 2 & 771 & $365 \cdot 17$ \\
\hline$p x$ & $s h v \mathrm{~L}$ & & Oregon & $\begin{array}{r}69 \\
69\end{array}$ & 255 & 300 & 3 & 627 & 390.48 \\
\hline sho $\mathrm{L}$ & $p x$ & Oregon & $p x$ & 134 & 249 & 349 & 0 & 732 & 343.87 \\
\hline
\end{tabular}

up of 707 flies of which 504 possessed extra vein material. The comparable cross of shv "short" to $p x$ produced only 8 extra vein carriers in an $\mathrm{F}_{1}$ of 753 flies. Again this difference between short and long lines is highly significant, demonstrating that selection for increased expression can increase the degree of dominance.

\section{Discussion}

Dominance, in this context, is a function not of a character or gene but of developmental relationships within an internal environment closely controlled or modified by the genetic background and external environmental factors (Burdick, 1951; Robertson, 1961; House, 1963). Dominance relationships are occasionally altered by the presence of one or more additional mutants (for example, Green and Oliver, 1940a, 1940b), and instances involving wing venation are well known (House, 1952, 1953a, $1953 b, 1954,1959 a, 1959 b, 1961)$. In the experiments described in this paper, double vein mutants allowed comparatively small changes in dominance relationships to be recognised. Canalisation was not investigated though this may be another important facet of dominance evolution (Rendel, $1959 a, 1959 b, 1967)$. The wild type remained dominant in single mutant tests, except that involving the development of semi-dominance in the 
veinlet "short" line, described above. It is realised, therefore, that these data only provide a demonstration of partial dominance arising through homozygote selection.

Fisher (1930) was the first to advance the idea that dominance is modifiable through selection. He proposed that a beneficial allele evolves dominance in response to the occurrence of deleterious allelic mutations, for, since in the absence of dominance each deleterious mutant produces a deleterious heterozygote, modifying factors that reduce the deleterious effects of the mutant in heterozygotes will be favoured by selection. A newly occurring mutant will appear first as a heterozygote, and homozygotes will not occur in high enough proportions for selection to influence them directly until the mutant frequency has risen considerably, except of course in the case of sex-linkage. For this reason most theories of the evolution of dominance discount the importance of selection in the homozygote, concentrating experimental work on selection of the heterozygotes (see, for example, Dunn and Landauer, 1934, 1936; Ford, 1940; Fisher and Holt, 1944).

There are at least two instances, however, in which selection in the homozygote should be considered. The first is related to the evolution of mimicry as proposed by Sheppard (1962). The heterozygote will be the only mimetic form when the mutant first arises. If the mimetic form has an advantage over the cryptic form, however, the new allele will increase in frequency until the advantage of the heterozygote is balanced by the disadvantage of the homozygote. From this point both the homozygotes and heterozygotes of the mimetic form will evolve toward the model and dominance will be attained. The theory thus requires homozygote selection to contribute to the evolution of dominance. The present study verifies that this expectation is reasonable.

The second instance is directly related to all theories in which the selection of the heterozygote is invoked. By Fisherian selection, because deleterious mutants are occurring repeatedly in the population as heterozygotes, modifiers are selected that reduce and eliminate heterozygote expression, thereby making these mutants recessive. If a condition, such as melanism, which has been deleterious during the early history of the species now becomes beneficial, how is it possible to select modifiers that reverse the established dominance relationships if dominance is complete. In other words, Fisherian selection cannot begin unless the newly beneficial heterozygote is distinguishable from the dominant homozygote. There are, however, at least three ways around this difficulty. First, evolutionary change might have to await new modifier mutants. Second, change might only be expected from genes for which complete dominance had not evolved. These alternatives place large restrictions on the flexibility of the evolutionary process. Third, enhancement of expression in the rare homozygote could give rise to some degree of heterozygote expression. As soon as the heterozygote can be distinguished from the dominant homozygote, that is, as soon as it becomes semi-dominant, dominance modification may proceed along any or all of the lines proposed by the major theories in the field.

That expression modifiers may affect the degree of dominance suggests a well-integrated relationship between dominance and expressivity of the phenotype. This is not meant to suggest that all modifiers of dominance necessarily modify expression. Goldschmidt and Hoener (1937) and Blanc (1946), working with Drosophila melanogaster, found that vestigial dominigenes 

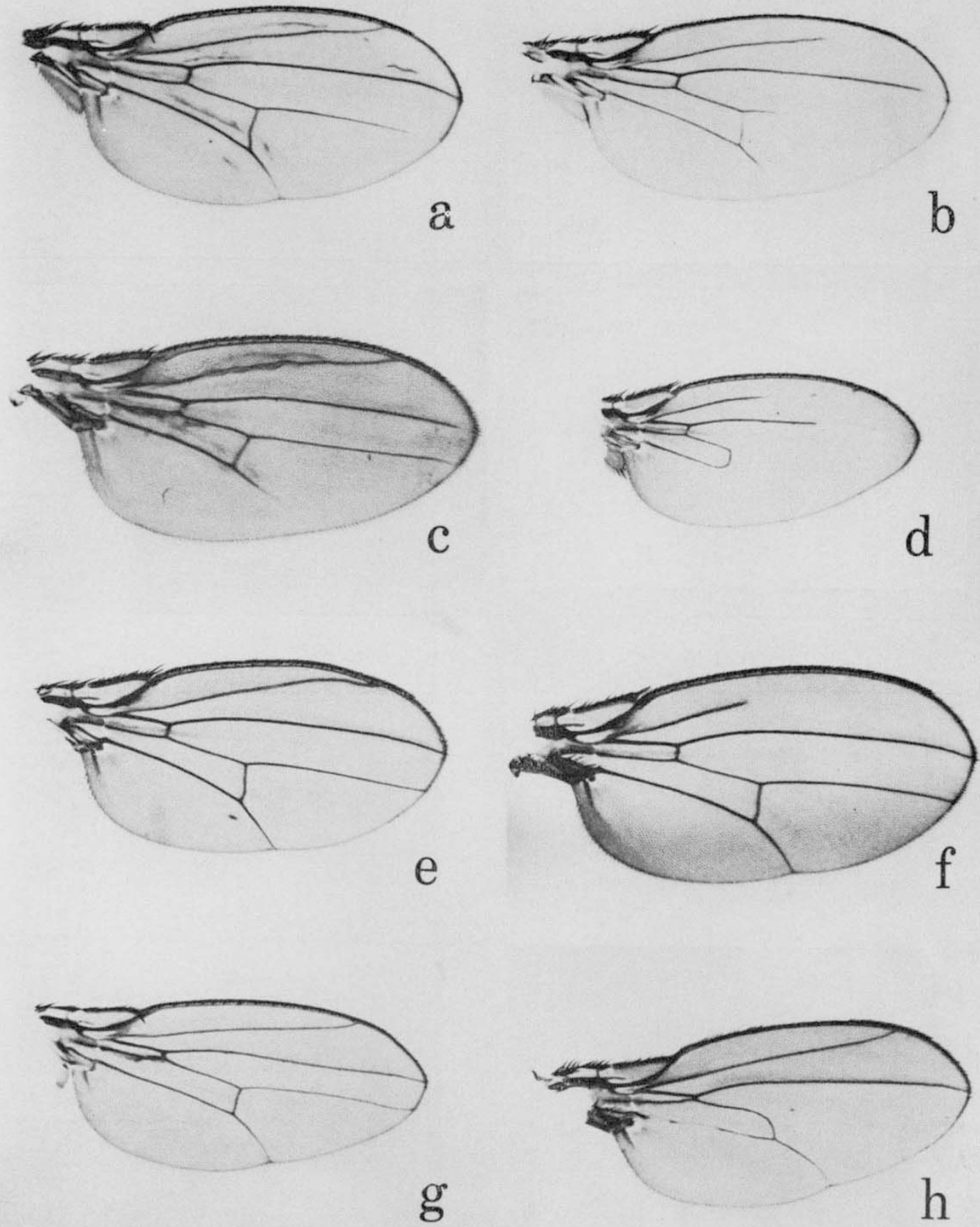

Wing vein mutants of Drosophila melanogaster selected for decreased (left) and increased (right) expression: $(a, b)$ short vein, shv; $(c, d)$ veinlet, $v e ;(e, f)$ radius incompletus, $r i$; $(g, h)$ cubitus in terruptus, $c i$. 
Plate II

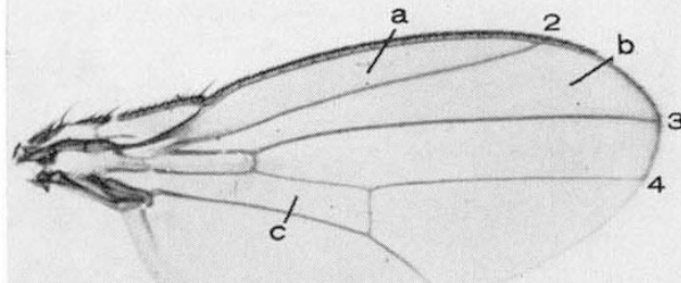

5

a

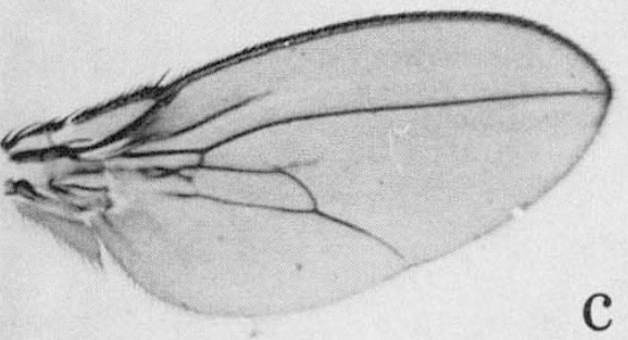

$\mathrm{C}$
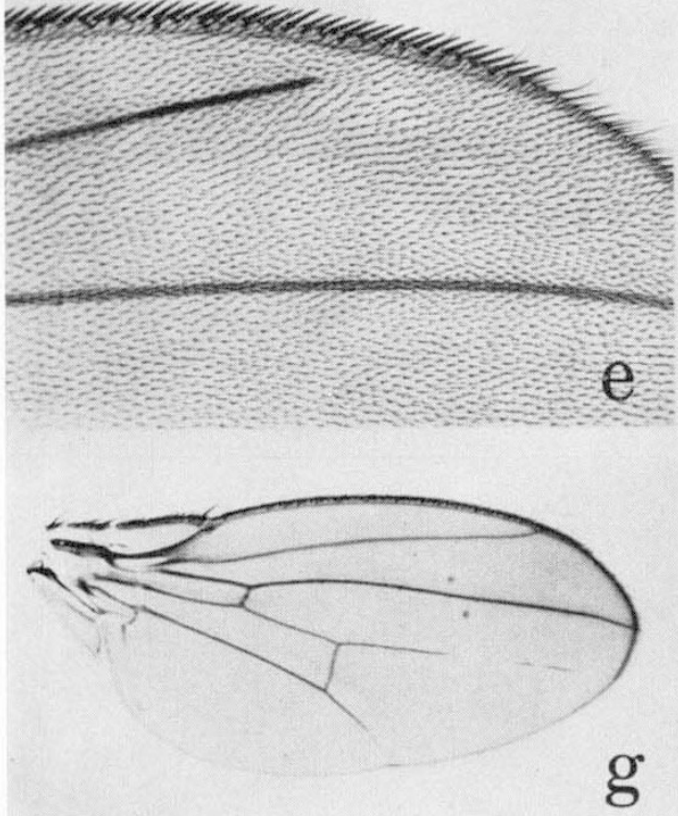

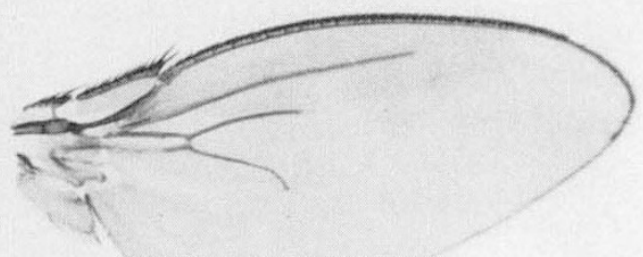

b

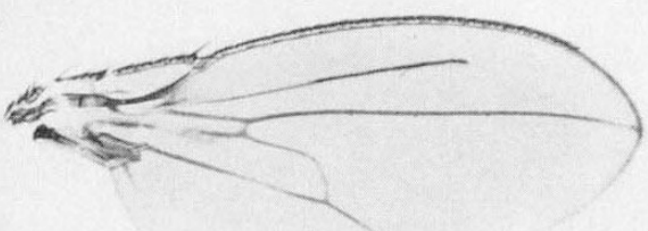

d
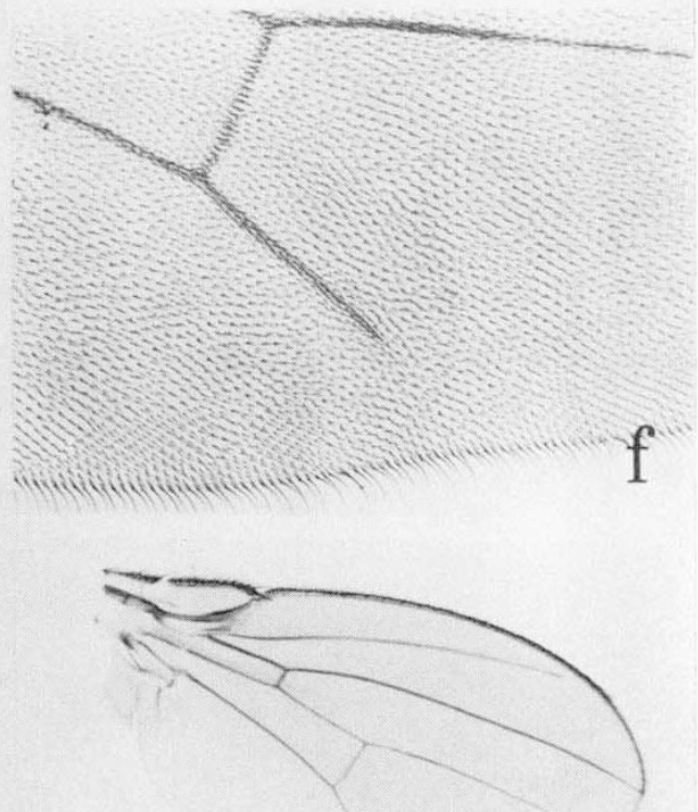

$\mathrm{h}$

Wings of Drosophila melanogaster: (a) wild type with the long vein designations indicated at the outer margin, marginal cell ("a "), submarginal cell ("b"), and discal cell ("c"); $(b-d)$ double homozygotes ve/ve; $c i / c i, r i / r i ; c i / c i$, and shv/shv; ci/ci; (e) vein L2 not joining margin; $(f)$ vein L5 shortened; $(g)$ vein L4 broken and L5 shortened from cross of shv short $\times v e$ short; $(h)$ veins L2 and L5 shortened from cross of $r i$ short $\times v e$ short. 
increased notching in both the heterozygote and homozygote of other alleles, though Dunn (1940) showed that the degree of dominance modification of the short-tail mutant in mice depended upon the stock to which it was outcrossed, while there was no apparent modification of the homozygote phenotype. Nor is it suggested that all modifiers of expression necessarily also effect dominance, but rather that such modification is a firm possibility and that homozygote selection should be investigated rather more fully in experimental or theoretical approaches to the question of the evolution of dominance.

\section{SUMmary}

1. Four mutants affecting wing vein length in Drosophila melanogaster were selected for long (reduced) expression and for short (increased) expression. At generation S-9, when significant differences in expression had been achieved in each mutant, double mutant heterozygotes were made by crossing together reduced-expression lines in all combinations. Similar double mutant heterozygotes were also produced for increased-expression lines. The increased-expression lines produced a highly significant excess of vein gaps in the $F_{1}$.

2. Lines selected for long (reduced) expression of the mutant short vein produced a highly significant excess of extra vein material when doubly heterozygous with the mutant plexus.

3. Selection in the homozygote produced semi-dominance in the third chromosome mutant veinlet.

4. The occurrence of vein defects in $F_{1}$ heterozygotes illustrates the alteration of dominance relationships through selection in the homozygote and demonstrates that some expression modifiers may also be dominance modifiers. Some applications to the theory of the evolution of dominance were briefly considered.

Acknowledgments.-We would like to thank Dr Peter O'Donald for reading the manuscript and Mr Brian Curtis and Mrs Christine Finnegan for preparing the photographs. J. N. T. gratefully acknowledges the support of a Marshall Scholarship.

\section{REFERENCES}

BLANC, R. 1946. Dominigenes of the vestigial series in Drosophila melanogaster. Genetics, 31, 395-420.

BODMER, W. F. 1960. Interaction of modifiers: The effect of pallid and fidget on polydactyly in the mouse. Heredity, 14, 445-448.

BURDICK, A. B. 1951. Dominance as function of within organism environment in kernelrow number in maize (Zea mays L.). Genetics, 36, 652-666.

CLARKE, G. A., AND SHEPPARD, P. M. 1962. Disruptive selection and its effect on a metrical character in the butterfly Papilio dardanus. Evolution, 16, 214-226.

DUN, L. c. 1940. Changes of dominance in the house mouse. Genetics, 25, 116.

DUNN, L. C., AND LANDAUER, w. 1934. The genetics of the rumpless fowl with evidence of a case of changing dominance. 7. Genet., 29, 217-243.

DUNN, L. C., AND LANDAUER, w. 1936. Further data on genetic modification of rumplessness in the fowl. 7. Genet., 33, 401-405.

FISHER, R. A. 1928. The possible modification of the response of the wild type to recurrent mutation. Am. Naturalist, 62, 115-126.

FISHER, R. A. 1935. Dominance in poultry. Trans. Roy. Soc., Lond., B, 225, 195-226.

FISHER, R. A. 1930. The Genetical Theory of Natural Selection. Clarendon Press, Oxford.

FISHER, R. A. 1931. The evolution of dominance. Biol. Rev., 6, 345-368. 
FISHER, R. A., AND HOLT, s. B. 1944. The experimental modification of dominance in Danforth's short-tailed mutant mice. Ann. Eugenics, Lond., 12, 102-120.

FORD, E. B. 1940. Genetic research in the Lepidoptera. Ann. Eugenics, Lond., 10, 227-252.

FORD, E. B. 1971. Ecological Genetics, Third Edition. Chapman and Hall, London.

GoldSCHMIDT, R., AND hOENER, E. 1937. Dominigenes and vg allelomorphs. Univ. Calif. Publ. Zool., 41, 297-312.

GREEN, M. M., AND OLIVER, C. P. 1940a. The action of temperature and of non-allelic mutants upon heterozygous vestigial in Drosophila melanogaster. Genetics, 25, 119-120.

GREEN, M. M., AND OLIVER, C. P. 1940 b. The action of certain mutants upon the penetrance of heterozygous vestigial wing in Drosophila melanogaster. Genetics, 25, 584-592.

HOUSE, v. L. 1952. The interaction of some mutants affecting venation in Drosophila melanogaster. Genetics, 37, 591-592.

HOUSE, v. L. 1953a. The interaction of mutants affecting venation in Drosophila melanogaster. I. Interaction of Hairless, engrailed, and cubitus interruptus. Genetics, 38, 199-215.

HOUSE, V. L. 1953b. The interaction of mutants affecting venation in Drosophila melanogaster. II. Additive and pattern effects of combinations of Hairless, engrailed, and cubitus interruptus. Genetics, 38, 309-327.

HOUSE, v. L. 1954. The interaction of mutants affecting venation in Drosophila melanogaster. III. The interaction at various temperatures of Hairless, engrailed, and cubitus interruptus 7. Exp. Zool., 127, 53-74.

HOUSE, v. L. 1959a. The interaction of Hairless and engrailed with cubitus-interruptusdominant and cubitus-interruptus-Wallace in Drosophila melanogaster. Genetics, 44, 516.

HOUSE, v. L. 1959b. A comparison of gene expression at the Hairless and Abruptex loci in Drosophila melanogaster. Anat. Rec., 134, 581-582.

HOUSE, v. L. 1961. Mutant effects in multiple heterozygotes of recessive venation mutants in Drosophila melanogaster. Genetics, 46, 871.

House, v. L. 1963. The effect of temperature on phenotypic expression and dominance relationships relative to two inbred lines of Drosophila melanogaster homozygous for the cubitus interruptus gene. Genetics, 48, 893-894.

LINDSLEY, D. L., AND GRELL, E. H. 1967. Genetic Variations of Drosophila melanogaster. Carnegie Institution of Washington Publ. No. 627.

o'donald, p. 1968. Models of the evolution of dominance. Proc. Roy. Soc., Lond., B, 171, 127-143.

OHH, B. K., AND SHELDON, B. L. 1970. Selection for dominance of Hairy-wing ( $H w)$ in Drosophila melanogaster. I. Dominance at different levels of phenotype. Genetics, 66, 517-540.

PARSONS, P. A., AND BODMER, W. F. 1961. The evolution of overdominance: Natural selection and heterozygote advantage. Nature, Lond., 190, 7-12.

RENDEL, J. M. 1959a. Variation and dominance at the scute locus in Drosophila melanogaster. Austr. 7. Biol. Sci., 12, 524-533.

RENDEL, J. M. 1959b. Evolution of dominance. In The Evolution of Living Organisms. Symposium of Royal Society of Victoria, Melbourne.

RENDEL, J. M. 1967. Canalisation and Gene Control. Academic Press, London.

ROBERTSON, F. w. 1961. The ecological genetics of growth in Drosophila. 4. The influence of larval nutrition on the manifestation of dominance. Genet. Res., Camb., 2, 346-360.

Sheppard, P. м. 1961. Natural Selection and Heredity. Hutchinson, London.

SHEPPARD, P. M. Some contributions to population genetics resulting from the study of the Lepidoptera. Adv. Genet., 10, 165-216.

SHEPPARD, P. M. 1962. Some aspects of the geography, genetics, and taxonomy of a butterfly. Systematics Association Publ. No. 4, 135-152. 